\title{
EL PABELLÓN DE VENEZUELA EN LA EXPO'92. UNA ESTRUCTURA DESPLEGABLE EN DURALUMINIO
}

\author{
(THE PAVILLION OF VENEZUELA AT THE EXPO' 92. \\ A FOLDING ALLUMINIUM STRUCTURE)
}

\author{
Carlos Hernández, máster en arquitectura por el MIT
}

Félix Escrig, Dr. arquitecto

E.T.S. de Arquitectura de Sevilla.

Fecha de recepción: 26 - III - 93

ESPAÑA

\section{RESUMEN}

Es la primera vez que una estructura extendible de aluminio es construida para uso arquitectónico. En este caso, un modelo basado en la desplegabilidad a través de unn sistema de acordeón fue concebido para cubrir un espacio de $30 \times 20 \mathrm{~m}^{2}$. El montaje se hizo en sólo unas horas, e igualmente el desmantelamiento, plegando la estructura para su futuro uso en otro sitio. El articulo explica las características de la obra con dibujos y fotos que muestran su comportamiento.

\section{SUMMARY}

It is the first time that an expandable structure made of alluminium is built for architectural use. In this case, a model based on accordion-like folding system is conceived to cover a span of $30 \times 20 \mathrm{sqm}$. The setting up was done in only a feu hours and folding uas also possible to reuse the structure in another place. Characteristics of this structure are explained with some drawings and pictures to shou' its behaviour.

\section{INTRODUCCIÓN}

La movilidad, facilidad de transporte y rapidez en el montaje han sido y son sueños permanentes de los constructores y los arquitectos. La gran servidumbre que el tiempo de construcción impone al proceso edificatorio ha convertido en una antigua aspiración la posesión de sistemas constructivos ligeros, intercambiables y portátiles.

En este sentido, los pueblos nómadas han aportado brillantes soluciones y las tiendas construidas con cubiertas textiles y estructuras vegetales han sido a la vez modelo de ligereza, adaptación al medio y portabilidad. También la movilidad de los ejércitos, condicionada muchas veces por el factor sorpresa y con exigencias de rápidos desplazamientos, han hecho concebir soluciones de alojamiento y protección consistentes en grandes tiendas. En las repre- sentaciones gráficas de la más remota antigüedad encontramos algunos de estos modelos. Y todavía en estos momentos uno de los requerimientos básicos ligados a estrategias de defensa es la erección de alojamientos y cubiertas de rápido montaje.

Pero no solamente para cerrar recintos, se han experimentado soluciones. Estructuras móviles se han necesitado para aprovechar la fuerza del viento, en barcos y molinos; para dar sombra en recintos arquitectónicos y urbanos, o para encerrar masas de aire que funcionan como estructura.

El Coliseo de Roma estaba dotado de un gigantesco entoldado que el personal de mantenimiento extendía y recogía a voluntad. El aerostastismo aprovecha la presión del aire caliente sobre un te⿳亠幺幺til, y esta misma presión es capaz de mantener eñ pie enormes cubiertas infladas. 
En ingeniería, puentes, pasarelas, mástiles y encofrados son un claro ejemplo de elementos que requieren rapidez de ejecución. El problema se ha hecho más acuciante en cuanto el implacable ritmo de las actividades actuales ha exigido que los procesos de montaje y desmontaje de elementos auxiliares de actividades sea mínimo. Ello ha dado origen a múltiples propuestas de sistemas modulares, sistemas inflables o mecanismos extensibles.

Igualmente la necesidad de construcción de grandes plataformas en el espacio extraterrestre, captadores de energía solar, antenas y estructuras soporte de módulos diversos ha obligado a experimentar propuestas radicalmente nuevas y, lo que en cierto modo es más importante, ha destinado financiación y medios para investigar en estos campos.

En el ámbito de las Estructuras móviles y de fácil montaje, hay que distinguir entre varios tipos de ellas que puedan cumplir un cometido similar y que tienen historia y funcionamiento bien diferenciados: Modulares, Textiles y Desplegables. Las modulares pueden ser incluso muy convencionales y aprovechan la facilidad de estar compuestas por un reducido número de elementos muy fáciles de conectar entre sí.

Las textiles deben su movilidad a su poco peso. La flexibilidad de sus elementos y la resistencia del material permiten omitir vigas, soportes y muros. Hay recintos textiles, tanto tensados como inflados que son enormemente sencillos en su concepción y montaje y se explica por ello que son de uso más antiguo. Los grandes avances de la tecnología los han hecho adecuados para grandes luces y para resolver algunos de los más ambiciosos proyectos.

\section{ESTRUCTURAS DESPLEGABLES}

Los desplegables son sistemas que pueden transportarse en paquetes en los que la específica conexión de los elementos tiene predeterminada la forma de desempaquetado. Son mecanismos que pueden adoptar una forma compacta o abrirse y convertirse en una gran superficie. Piénsese en una esponja o un muelle que comprimidos ocupan una mínima porción del espacio que ocuparán al liberarse. El hecho de ser mecanismos condiciona que estas estructuras deben bloquearse en algunas de sus posiciones extremas para que sean utilizables y no sigan moviéndose arbitrariamente. Es sobre estas estructuras sobre las que queremos hablar para introducir el Pabellón de Venezuela. Las estructuras desplegables a su vez pueden clasificarse en varios tipos basados en principios mecánicos distintos o construidos por elementos diferentes. Así tendremos "estructuras abisagradas" que están constituidas por paños planos que se abaten unos sobre otros, y "estructuras articuladas" que están formadas por barras que se conectan mediante rótulas unas con otras.

Las estructuras abisagradas pueden estar formadas por planos que aúnan rigidez y opacidad al mismo tiempo (Fig. 1) o por celosías planas de barras (Fig. 2).

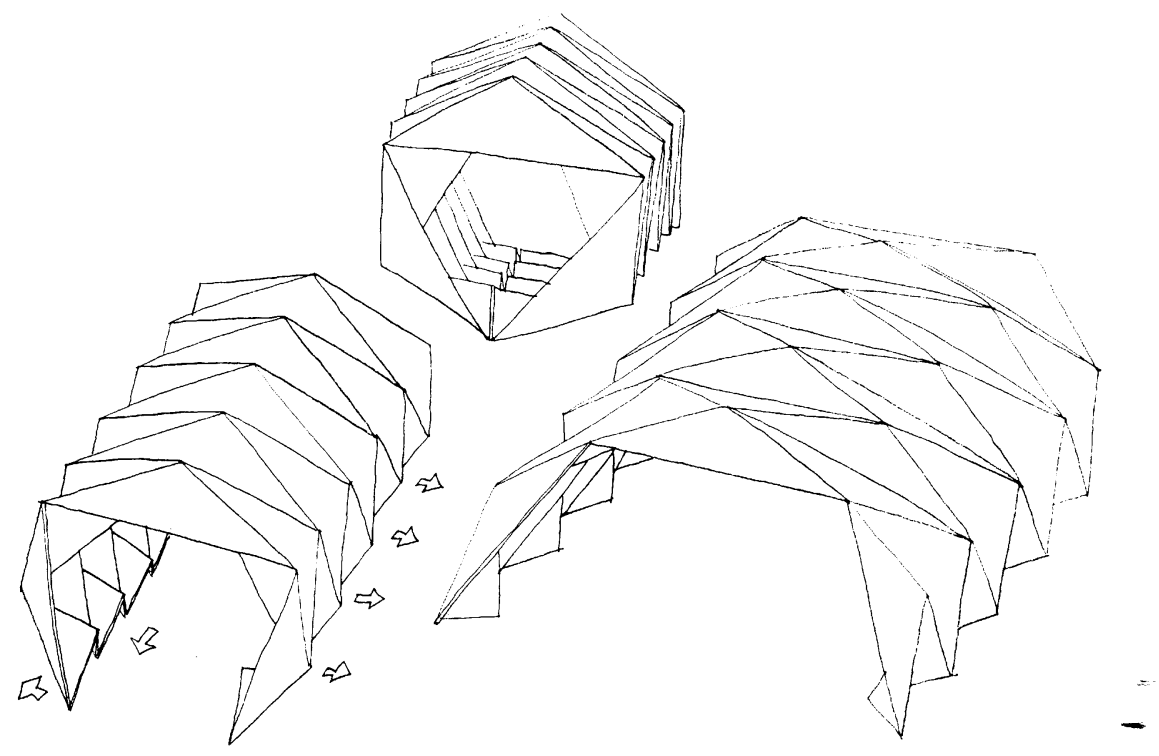

Fig. 1.- Propuesta de J.M. Prada para una desplegable de paneles. 

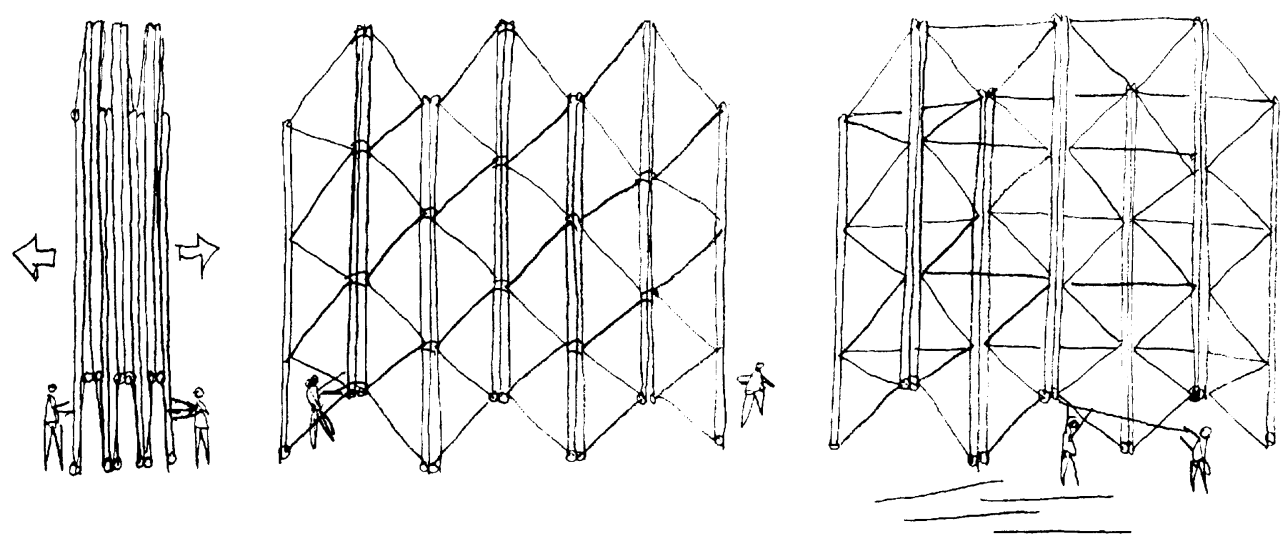

Fig. 2.- Desplegable de celosía arriostrada tras el despliegue.

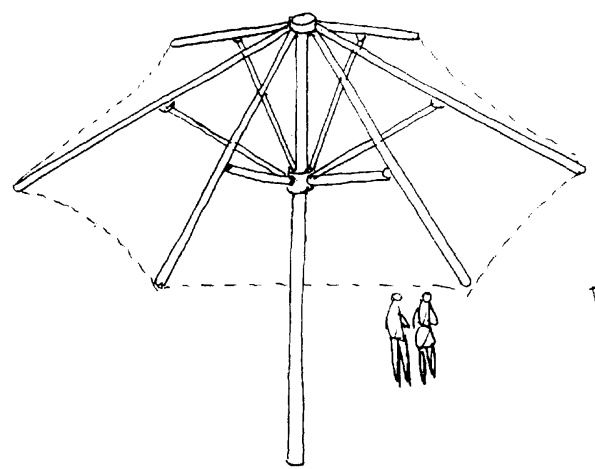

Fig. 3.- Estructura en paraguas.

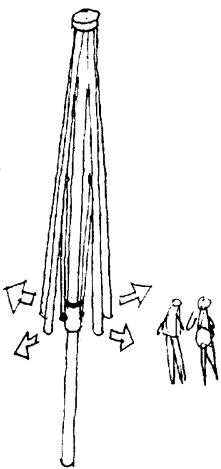

1.

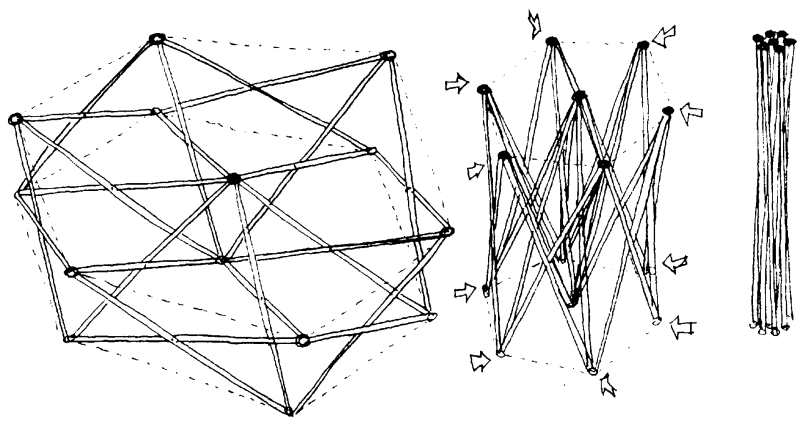

Fig. 4.- Desplegable de aspas
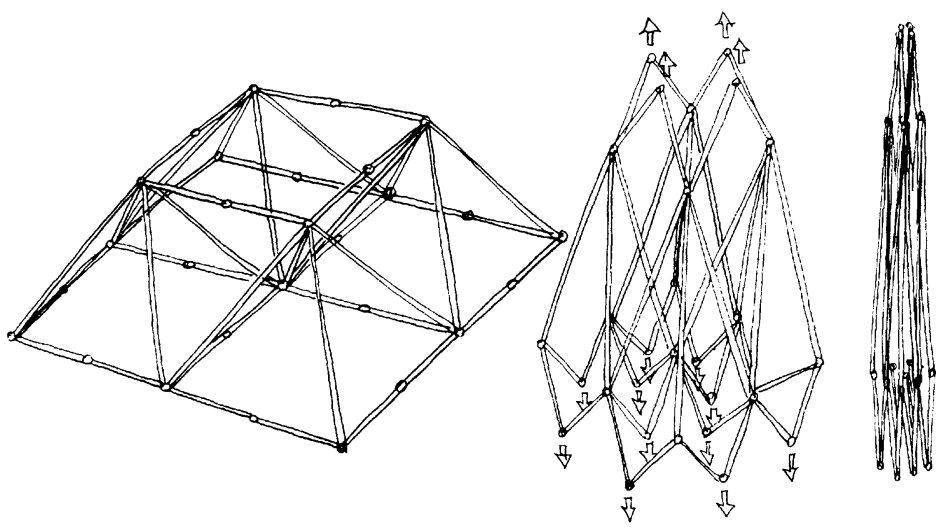

Fig. 5.- Desplegable de bielas.

Las estructuras articuladas están constituidas fundamentalmente por piezas rectas que forman el esqueleto sustentante del conjunto y que luego habrá que completar con paneles de cierre y elementos de cubierta. Eventualmente puede llevarlos incorporados. Estas estructuras articuladas a su vez pueden corresponderse a tres modelos básicos de funcionamiento: paraguas, tijeras y bielas.

En la Fig. 3 vemos un esquema simplificado de una estructura en paraguas que se basa en la posibilidad de movimiento basculante de unas barras situadas radialmente desde un pivote común. Los sistemas de erección y bloqueo pueden ser de distinta espe- cie. En el caso de la Fig. 3 son unos codales que convergen en un casquillo deslizante que puede bloquearse en las posiciones deseadas.

En la Fig. 4 vemos una composición de aspas o tijeras que, conectadas entre sí, dan unas posibilidades de crecimiento espacial sin restricciones. En la Fig. 5 una malla espacial semioctaédrica y por tanto rígida y estable puede comprimirse si disponemos de una articulación central en cada una de las barras de las capas superior e inferior. Estos cordones articulados son en realidad bielas y dejarán de serlo cuando se bloquee su articulación. 
En todos los casos, la posición inicial es la de un paquete compacto y la final la de una cubierta de gran desarrollo en relación al primer estado.

Las estructuras desplegables en general requieren tecnologías de cierta envergadura y materiales estructurales de gran resistencia y poco peso. Ello ha hecho que hasta muy recientemente no hubiera propuestas constructivas factibles. Concretamente es un español, Emilio Pérez Piñero, quien en los años 60 patenta algunas llamativas soluciones y con ello fomenta el interés y las posibilidades por el problema. Las agencias espaciales de diversos estados han ensayado también algunas soluciones, algunas de ellas puestas en práctica en el espacio exterior.

Entre los inventores que han trabajado el tema debemos destacar además del pionero citado, Pérez Piñero, a Buckmister Fuller, con grandes aportaciones conceptuales, Santiago Calatrava, con aplicaciones a la arquitectura móvil bien conocidas, y Zeigler, que ha desarrollado procesos industrializados de producción de mallas desplegables. Se cita como curiosidad sorprendente que ya Leonardo da Vinci en el Códice de Madrid aporta una estructura desplegable e intenta calcularla. Entre los grupos de Investigación que están publicando y aportando innovaciones sobre el tema debemos citar los de la Escuela de Arquitectura del MIT en Estados Unidos, con Zalewsky a la cabeza. Los del IDEC en Venezuela, con C.H. Hernández; los de La Coruña y Sevilla, con Pérez Valcarcel y Escrig dirigiéndolos; los de Cambridge en Inglaterra con Pellegrino; y otros de menor producción pero de aportaciones relevantes. Representantes de algunos de estos grupos nos hemos reunido en el Pabellón de Venezuela para hacer realidad un Proyecto Universal.

Obviamente, dependiendo del uso así será la tipología que pueda resultar más adecuada. En arquitectura necesitamos soluciones que sean fáciles de montar, sencillas de cerrar para crear recintos controlables con un determinado confort y desmontables con aprovechamiento total de las piezas y en tiempo breve; muy distinto a los que podemos necesitar por ejemplo en el espacio en donde la recuperabilidad queda descartada. Uno de los grandes condicionantes de la arquitectura es la reutilización y ello predetermina algunas de las soluciones entre las citadas.

\section{PLANTEAMIENTO ESTRUCTURAL}

Concretamente para desarrollar el Pabellón de
Venezuela los arquitectos venezolanos Enrique Hernández y Ralph Erminy, sus autores, estuvieron barajando varias alternativas que finalmente se decantaron en la utilización de una "estructura abisagrada con celosías planas abatibles". La experiencia del IDEC "Instituto de Desarrollo Experimental de la Construcción" de la Universidad Central de Venezuela en Caracas, hacía factible considerar otras propuestas, pero se eligió ésta por varias razones:

- Los requerimientos formales del Pabellón eran prioritarios a la imagen de la propia estructura, por lo que había que adaptarse a un diseño preconcebido.

- Debía resolverse con un mínimo de elementos componentes distintos, fueran éstos estructurales o de cerramiento.

- Debía tener un grado de rigidez suficiente como para ligar a los elementos soporte paneles acústicos y otros equipos necesarios para la Sala de Proyecciones.

- Debía resolverse con una estructura fácil de transportar, montar y desmontar por personal, llegado el caso, no especializado.

- Al predeterminarse su realización en aluminio estructural por la participación de una empresa del sector en la cooperación a la construcción debían utilizarse piezas especiales fabricadas por extrusión.

Por estas razones y, también, por un deseo de materializar una intención estructural previa se optó por la solución citada.

Los planos abisagrados utilizados estaban formados por vigas en celosía que daban rigidez estructural en su propio plano. Para garantizar la rigidez en cualquier otra dirección se añadirían unos codales transversales que atarían contra un despliegue adicional o contra un repliegue la estructura. Al mismo tiempo, estos codales mejoraban la rigidez del conjunto.

Esta estructura, cuya descripción se hace extensivamente en otro capítulo, tenía por objeto, con este concepto de desplegabilidad, el instalarse de una sola vez y en una sesión de montaje. No obstante, su gran tamaño, el hecho de que el sistema elegido da compacidad en una dirección pero no en la perpendicular, hicieron aconsejable partir el conjunto e instalarlo en dos sesiones. Aun así una de las partes tenía $38 \mathrm{~m}$ de longitud en dos tramos. La conexión de las piezas se hacía de un modo sumamente elemental mediante tornillos (Fig. 6). 


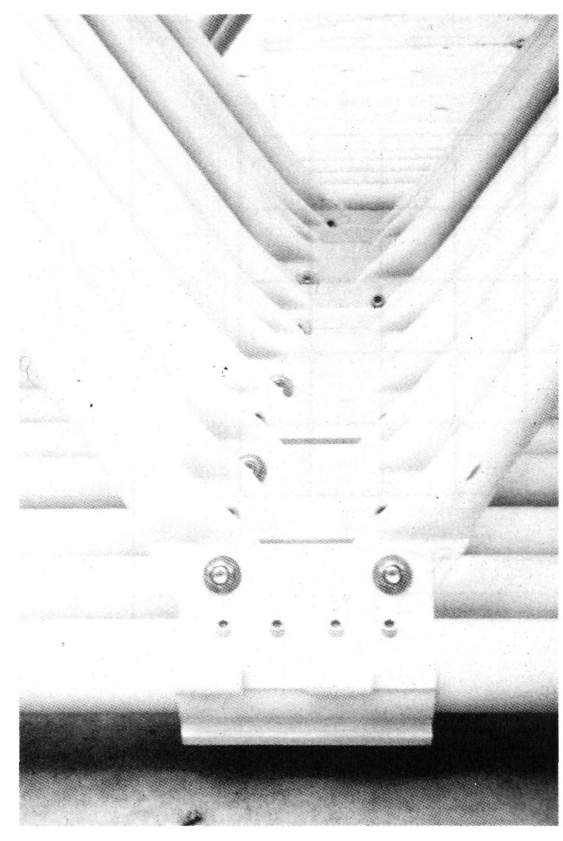

Fig. 6.- Conexiones de nudos con barras en el pabellón de Venezuela.

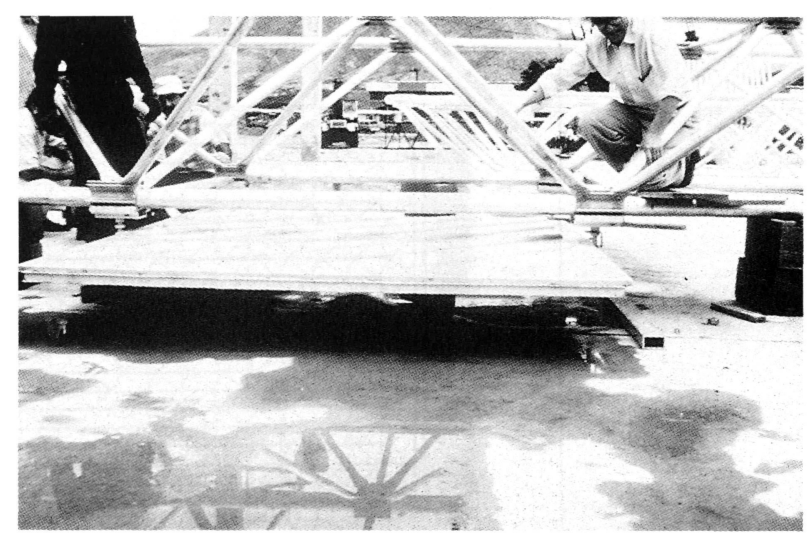

Fig. 7.- Conexión del cerramiento con la estructura.

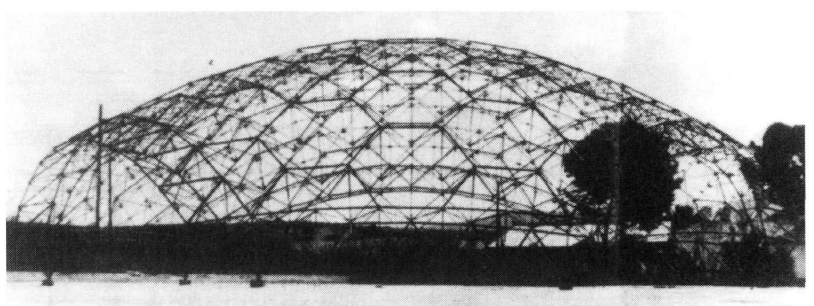

Fig. 8.- Cúpula desplegable de Emilio Pérez Piñero.

se barra a barra con facilidad por tener una ley de conexión sencilla y pocos elementos distintos. Una de sus obras más características es el Pabellón de EEUU en la EXPO de Montreal de 1967. Calatrava es un especialista de arquitectura móvil y sus propuestas desplegables todavía están en el papel. En este sentido su obra más singular son las puertas para la fábrica de Ernsting. Zeigler está trabajando en escalas reducidas y sus estructuras, hasta diez metros de luz, apenas pesan cincuenta kilos. Piñero en cambio construyó una cúpula de casi treinta metros de luz por fragmentos conectados entre sí y, pese a ser el primero, sigue siendo el más ambicioso en los planteamientos experimentados (Fig. 8).

En este contexto, e incluso incluyendo otras propuestas de los miembros autores del Pabellón de Venezuela, la estructura de éste es una aportación clave que abre fronteras a la arquitectura. Tanto por dimensiones $30 \times 20 \mathrm{~m}$ de planta, $19 \mathrm{~m}$ de altura y $7.500 \mathrm{~m}^{3}$ de volumen como por los materiales utilizados, aluminio aleación 6261 con resistencia a la rotura de $3.300 \mathrm{~kg} / \mathrm{cm}^{2}\left(3.000 \mathrm{~kg} / \mathrm{cm}^{2}\right.$ a la fluencia), por su peso de $10 \mathrm{t}$, con un material de $27 \mathrm{~kg} / \mathrm{m}^{3}$ de densidad, como por la sencillez de su montaje puede defenderse como un récord este por sus estructuras geodésicas que podían levantar- 


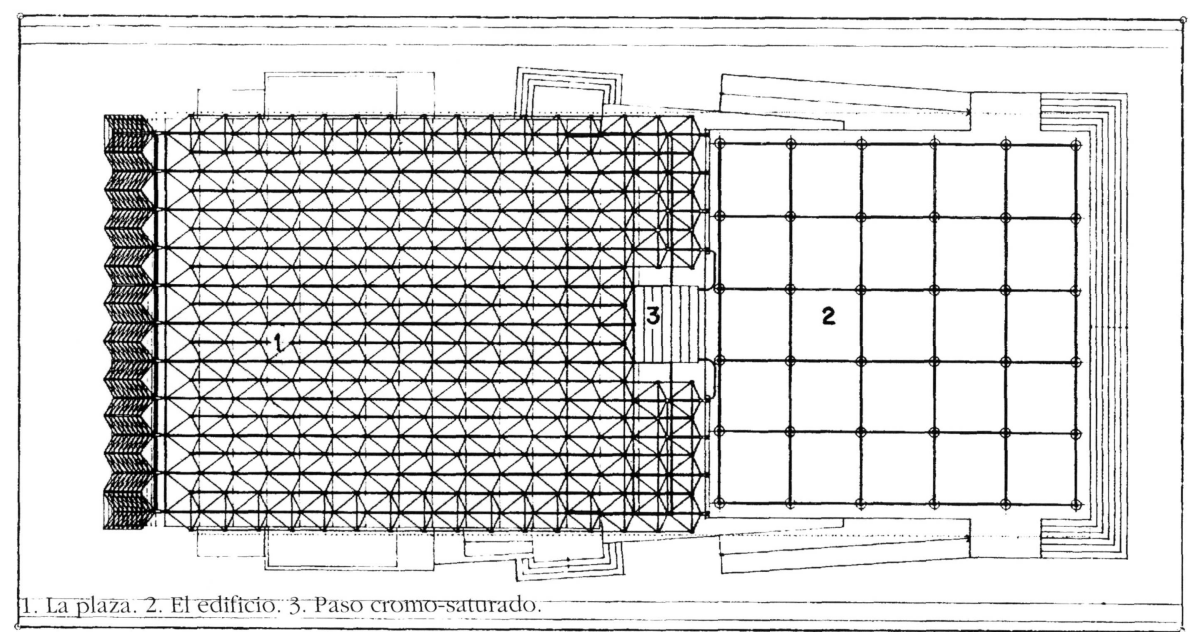

Fig. 9.- Planta de la estructura.

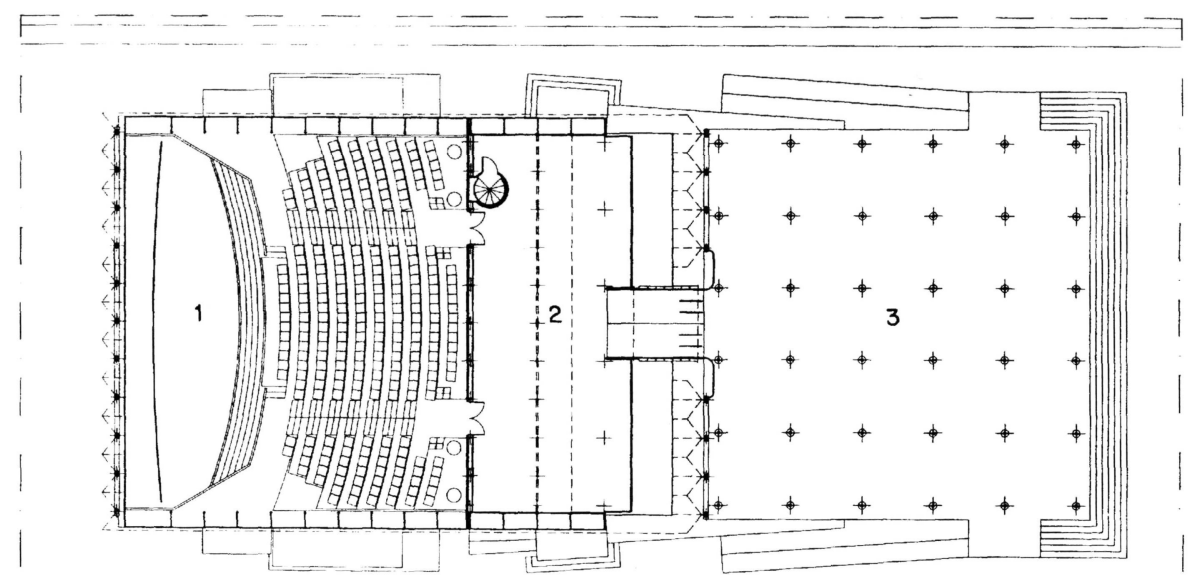

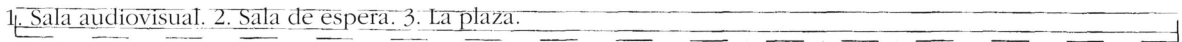
Fig. 10.- Planta de la plaza, del vestíbulo y sala de proyecciones.

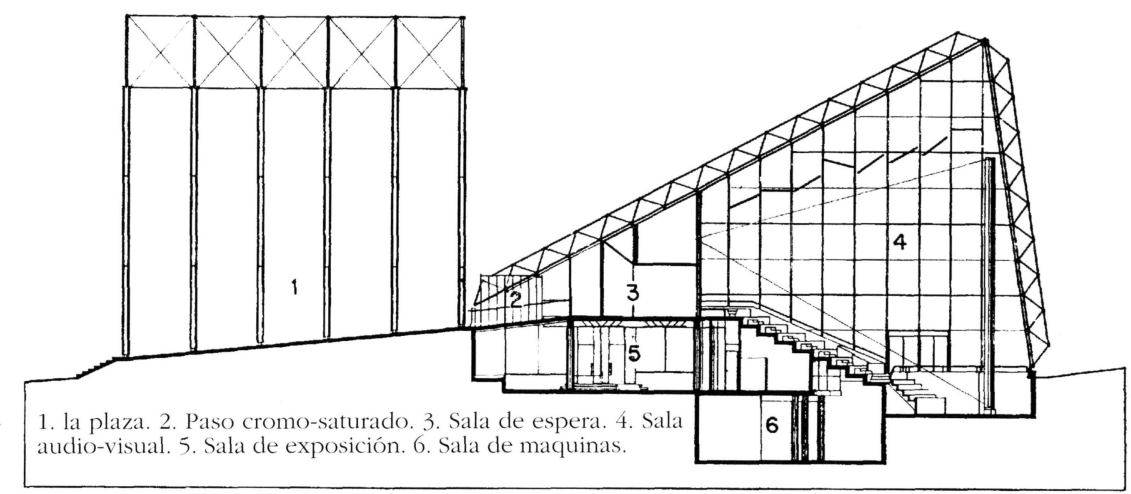

Fig. 11.- Sección longitudinal.

edificio singular y representativo. La topografía compleja de la arquitectura base, sobre la que debía asentarse por requerimientos funcionales, hicieron algo más difícil de lo deseable un montaje paradigmático. Pero también contaba como una virtud el haber sido capaz de asentarse sobre esa sección de varios niveles. El montaje de cada una de las dos partes de la estructura pudo concluirse en un sólo día y con el conjunto bien estribado ya se procedió a complementarlo con riostras y paneles. La confirmación de los objetivos propuestos se ha alcanzado no obstante cuando el edificio se ha desmontado y, retrocediendo los pasos de su instalación, ha regresado a su país de origen para ser de nuevo erigido. Cuando eso concluya se habrá cumplido una experiencia histórica. En las Figs. 9 a 12 se muestran las características generales del proyecto. 


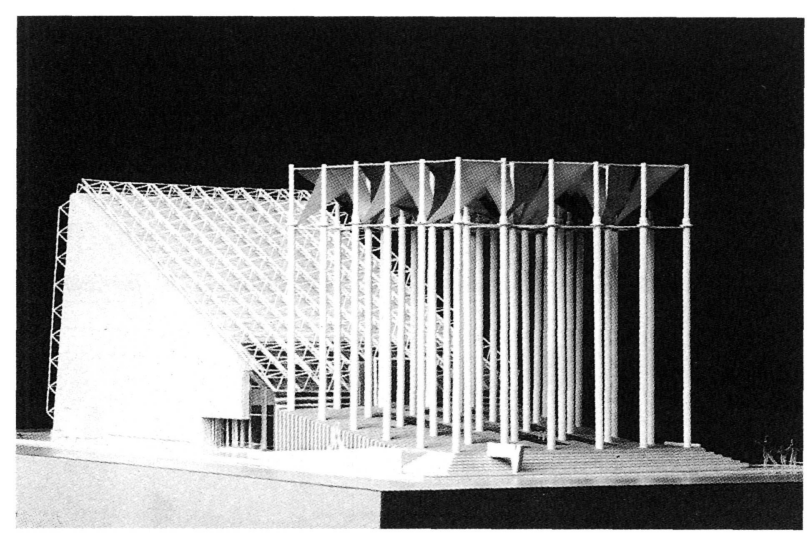

Fig. 12.- Maqueta de conjunto.

\section{DESCRIPCIÓN DE LA ESTRUCTURA Y MONTAJE}

La estructura está formada por dos partes que se transportan por separado.

La más pequeña de ellas está formada por 16 cerchas de $13 \mathrm{~m}$ y 6 de 9,20 $\mathrm{m}$ articuladas entre sí para formar planos plegados en acordeón según vimos en la Fig. 4 (Fig. 13). Esta estructura llegó a obra formando un paquete de $13 \times 2 \times 1,4$ metros.

La mayor está formada por dos bloques similares de 18,20 m cada uno, con 22 cerchas cada uno articuladas del mismo modo, y que se izó y desplegó antes de su colocación en la posición definitiva como se indica en las Figs. 14, 15 y 16.

Una vez desplegadas estas estructuras se atornillaron a la base de hormigón, se conectaron entre sí y se rigidizaron mediante la colocación de barras transversales que la convirtieron en una estructura espacial de módulo semioctaédrico. La Fig. 17 muestra la conexión de las barras transversales 07 sobre las cerchas principales 06. Al mismo tiempo se muestra cómo se conectó el panel del techo a los nudos de la capa inferior.

La Fig. 18 muestra el diseño del nudo tipo fabricado a partir de un perfil concebido expresamente y fabricado por extrusión. Este nudo está formado por dos piezas a través de las cuales pasa el perfil de aluminio de los cordones $\varnothing 72$ e. $3 \mathrm{~mm}$ articuladas entre sí y que una vez abierto se bloquea con una plaqueta que lo inmoviliza. Este nudo también está provisto de pestañas para conectar las barras diagonales $\varnothing 40$ e. $3 \mathrm{~mm}$ y transversales.

(c) Consejo Superior de Investigaciones Científicas Licencia Creative Commons 3.0 España (by-nc)

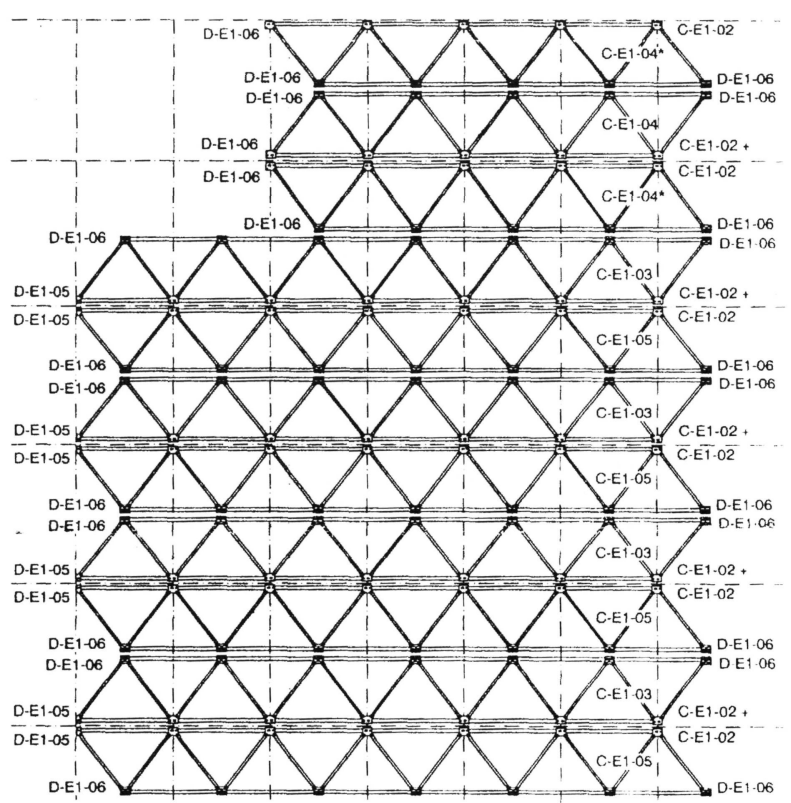

Fig. 13.- Despiece del tramo menor de estructura desplegable.

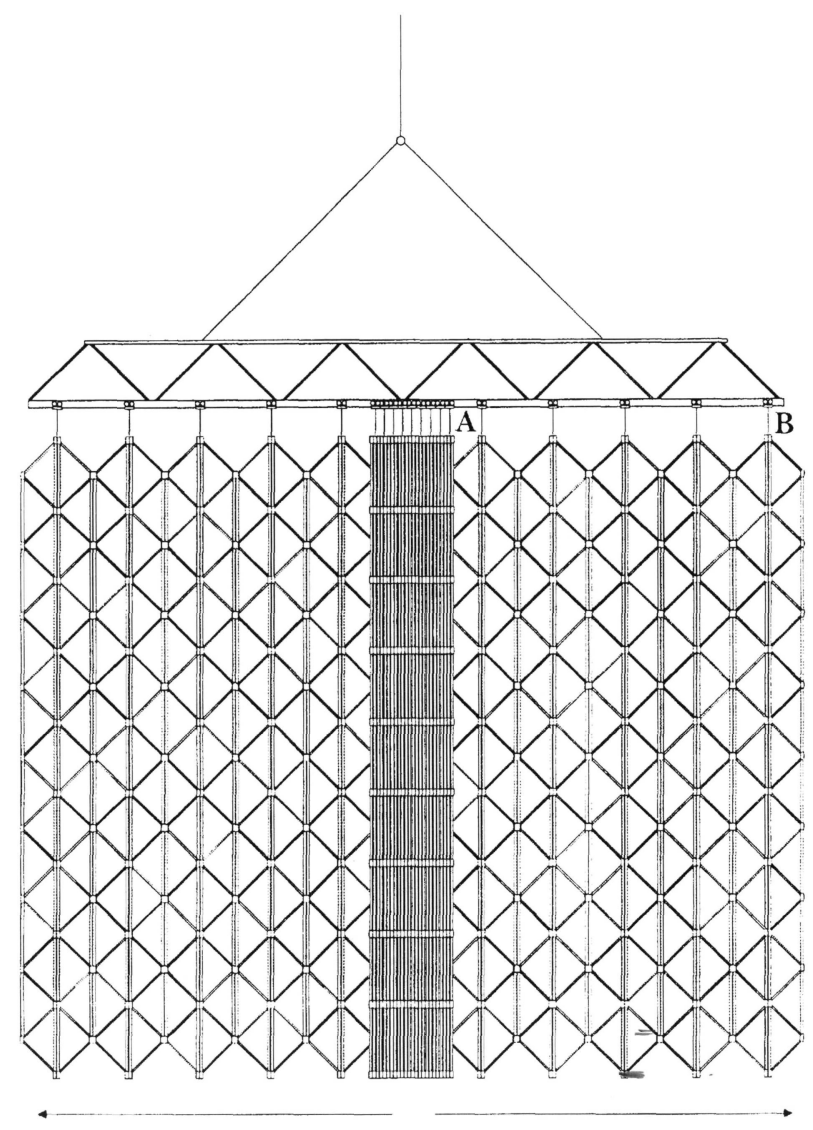

Fig. 14.- Despliegue del tramo mayor de la estructura desplegable. 


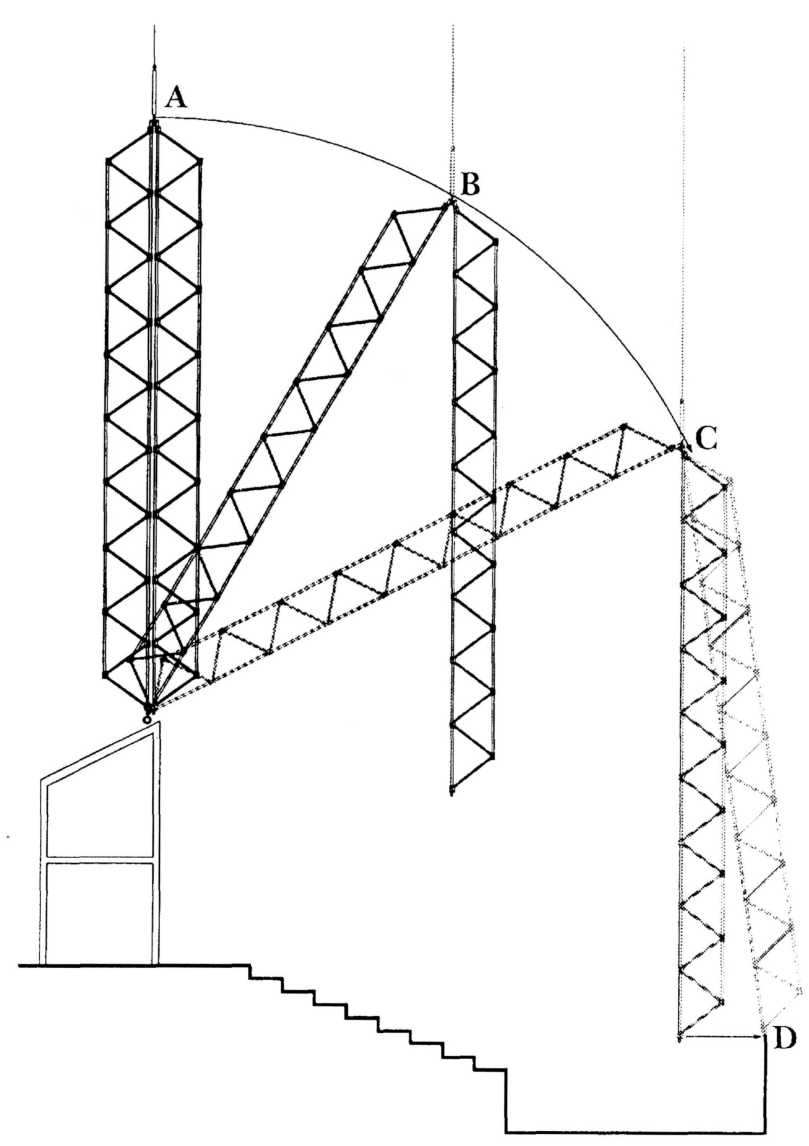

Fig. 15.- Modo de posicionar la estructura mayor.

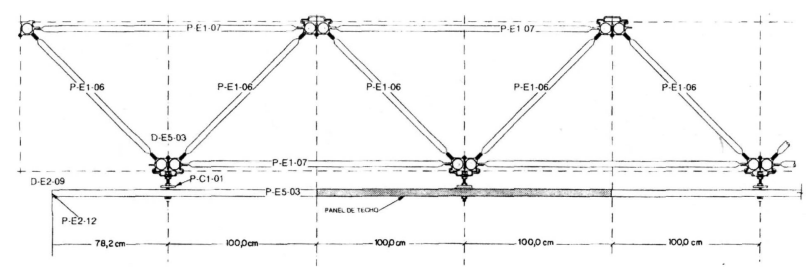

Fig. 17.- Esquema de conexión de la estructura a los paneles de cerramiento.

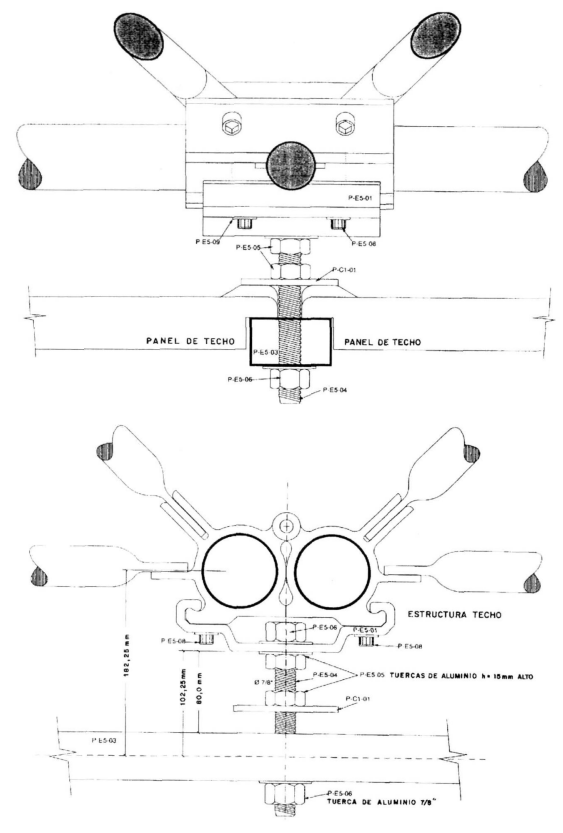

Fig. 18.- Detalle del nudo tipo articulado utilizado para resolver el plegado de la estructu-

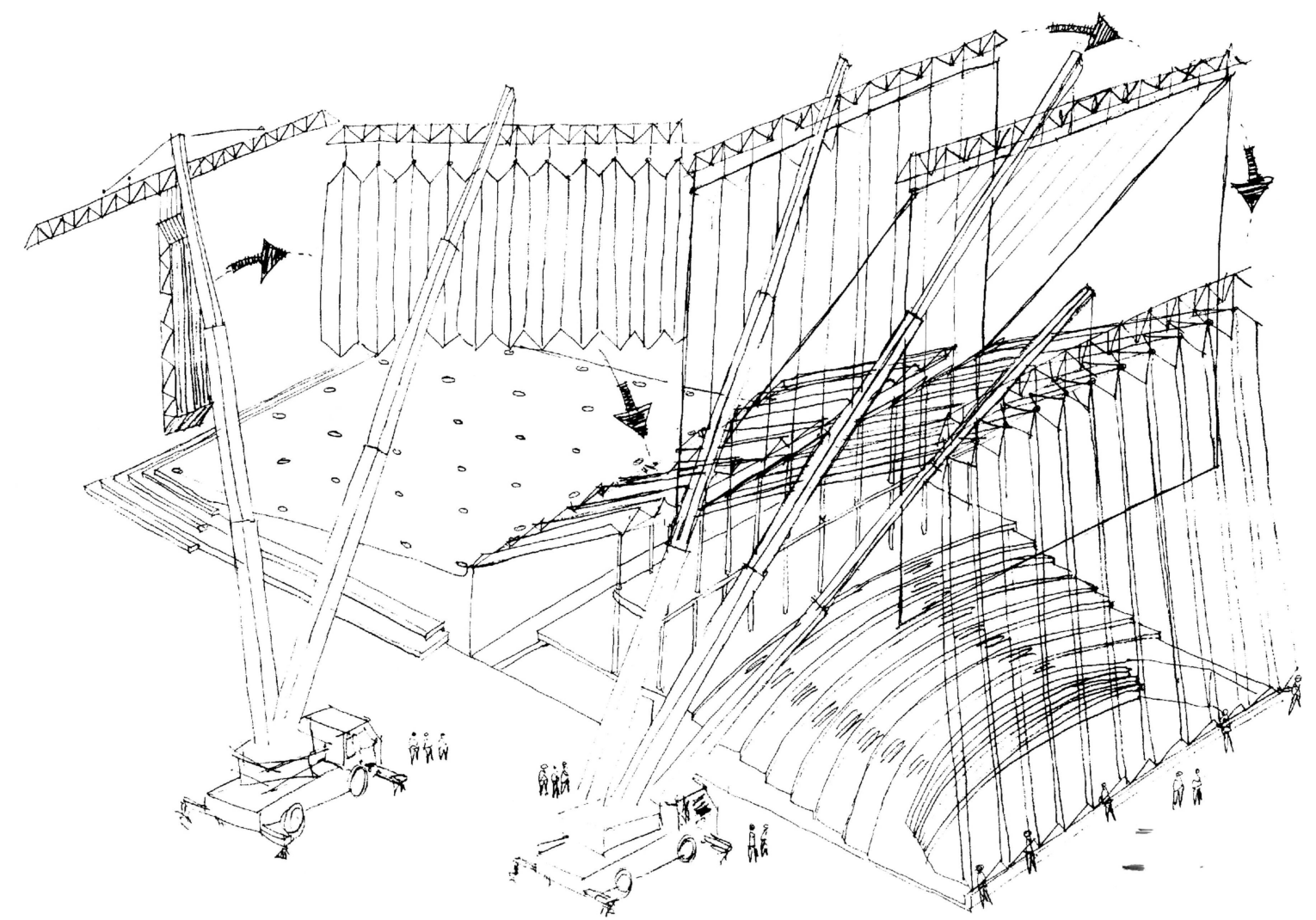

Fig. 16.- Esquema general de montaje de la estructura desplegable. 

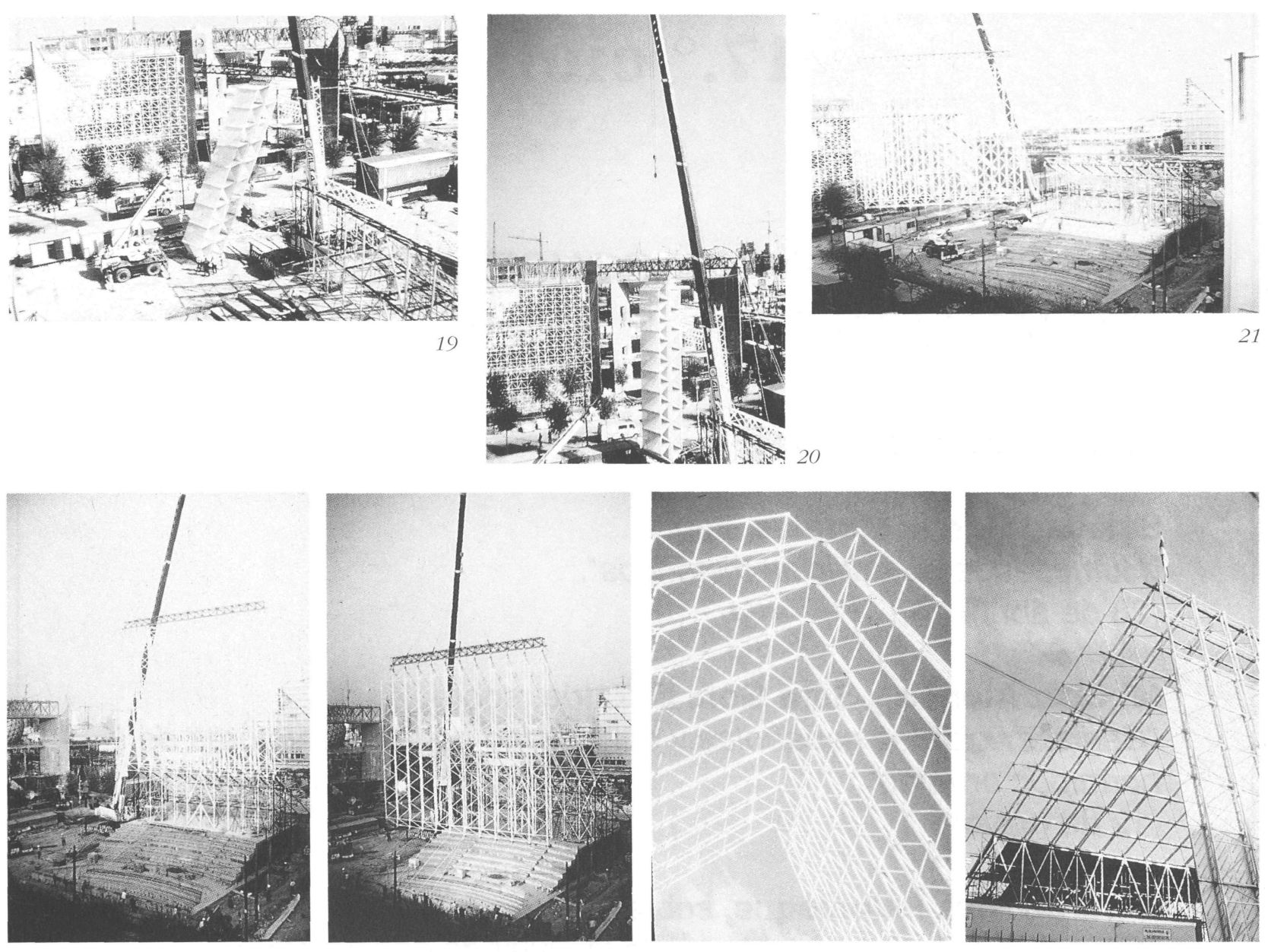

22

23

24

25

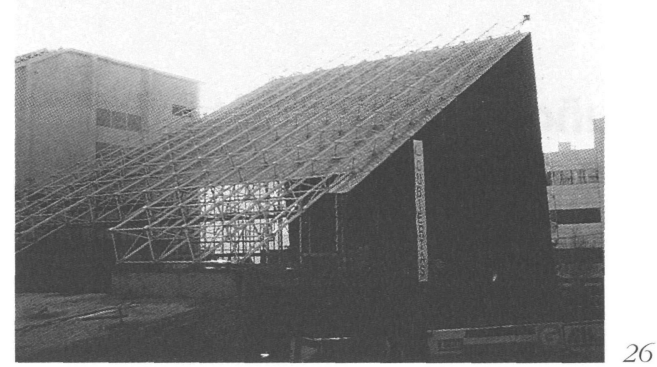

Figs. 19 a 26. Secuencia de montaje de la estructura desplegable.

Las Figs. 19 a 26 muestran distintas fases del montaje. El cuidado diseño, la exhaustiva definición del proyecto y la ejecución industrializada con una tecnología avanzada hicieron que todas las previsiones de montaje y posterior desmontaje resultaran enor- memente precisas y sin complicaciones.

El Pabellón de Venezuela en la EXPO`92 de Sevilla es un ejemplo imponente de lo avanzado de la industria del aluminio venezolano.

\section{Cuadro técnico}

\begin{tabular}{|ll|}
\hline Equipo de diseño-proyecto: & Mr. Carlos Hernández \\
Arq. Henrique Hernández & Supervisión técnica: \\
Arq Ralph Erminy & Arq. Félix Escrig \\
Arq. Marcel Erminy & Arq. Ricardo Huete \\
Estructura: & Arq. Téc. Feliciano Muñoz \\
Dr. Waclaw Zalewski & \\
\hline
\end{tabular}




\section{SEMINARIOS TORROJA'94 1\%. ${ }^{\circ}$ ciclo}

Tema:

\section{"TECNOLOGÍA DE LA CONSTRUCCIÓN Y DE SUS MATERIALES"}

Actuarán como ponentes:

Soledad García Morales, sobre:

"Humedades en edificios históricos".

(28 de abril).

Ma Cruz Alonso y Sara Goñi Elizalde, sobre:

"Cenizas volantes españolas y durabilidad de las estructuras de hormigón armado".

(12 de mayo).

Gerardo Ruiz Palomeque, sobre:

"Situación actual del problema de la vivienda en Cuba".

(26 de mayo).

José Alejandro Muñoz Robleño, sobre:

"El refuerzo de las estructuras de hormigón y su patología".

(9 de junio).

Pablo Zuloaga Lalana, sobre:

"Almacenamiento de residuos radiactivos. El Centro de El

Cabril".

(23 de junio).

Estos Seminarios tendrán lugar en el

Aula Eduardo Torroja,

a las 12 horas

La asistencia será gratuita

\section{ICCET}

CSIC : 


\title{
publicación del CSIC/ICCET
}

Razón y Ser de los tipos estructurales alcanza en la Colección "Textos Universitarios" su séptima edición. Las anteriores fueron publicadas sucesivamente por el Consejo Superior de
Investigaciones

Científicas a través del Instituto de Ciencias de la Construcción "Eduardo Torroja", que lleva el nombre de su autor. El prestigio internacional de Eduardo Torroja Miret

\section{TEXTOS UNIVERSITARIOS}

\author{
EDUARDO TORROJA MIRET
}

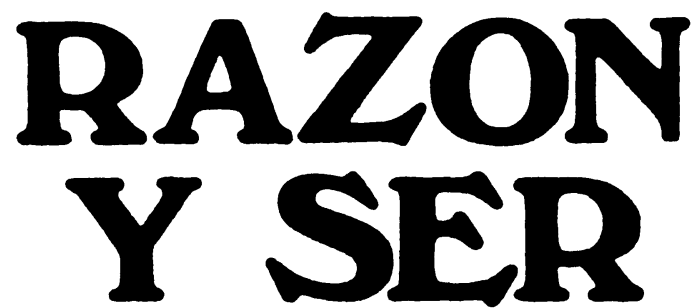

DE LOS TIPOS ESTRUCTURALES

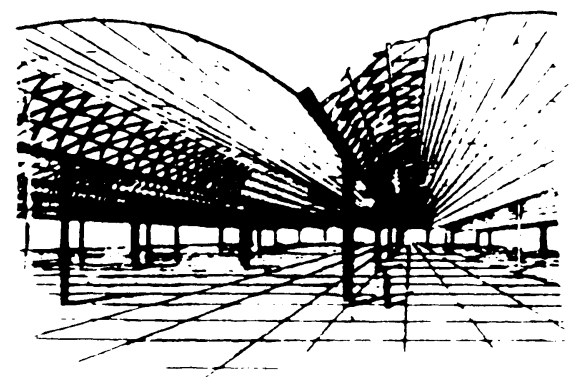

\section{Consejo Superior de Investigaciones Cientificas}

dentro de la Ingeniería como creador e innovador en el campo de las estructuras, es bien conocido. Esta capacidad excepcional de creación, interpretación $e$ innovación queda perfectamente reflejada en este libro.

Obra fundamental de notable contenido formativo y didáctico para los estudiantes y estudiosos de la Arquitectura y la Ingeniería, Razón y Ser mantiene, a pesar del tiempo transcurrido desde su edición original, plena vigencia.

Este hecho y la demanda continua de ejemplares, dentro y fuera de España, nos ha animado a su reedición en una colección que como "Textos Universitarios" se plantea llevar a sus lectores obras fundamentales de permanente demanda, $y$ nuevas aportaciones igualmente importantes tanto por la novedad de sus contenidos, como por la solidez y actualidad de los mismos.

Finalmente, esta nueva edición de Razón y Ser mejora, a nuestro juicio, la calidad editorial y presentación de las anteriores, y esperamos obtenga la misma acogida de las que la precedieron.

Madrid, octubre 1991 


\section{LA FÁBRICA ARMADA = MATERIAL COMPUESTO}

Seguridad y economía en la albañilería
Josep $\mathbf{M}^{\mathbf{a}}$ Adell, Profesor Dr. Arquitecto
Dpto. Construcción y Tecnologia Arquitectónicas - Escuela T. S. Arquitectura - Universidad Politecnica Madrid
La Arquitectura, tradicionalmente ha tenido en la fábrica uno de los mejores exponentes de la construcción. Los
materiales de fábrica actuales, han mejorado sus cualidades para cumplir su cometido de la forma más idónea posible. Por
otra parte, el acero que se aplica en muchos capítulos de la edificación, puede complementar perfectamente la albañilería.
El armado, con su ductilidad, evita los problemas derivados de la habitual rigidez de las fábricas, optimizando la aplicación
de sus materiales, en benefico de una nueva Arquitectura de fábrica segura y económica.

Para armar adecuadamente cualquier tipo de fábrica siguiendo la técnica de la albañilería, es preciso prefabricar una estructura de alambres de reducido grosor que quepa en el tendel. La armadura de tendel "Murfor" de Bekaert, es la armadura europea para la albañilería. Consiste en dos alambres paralelos, distanciados entre sí mediante un alambre diagonal en zig-zag soldado en su mismo plano, constituyendo la triangulación de una cercha. Tiene diversas protecciones frente a la corrosión (Zinc-Epoxi-Inox.) y existen anchos apropiados para cada material y tipo de fábrica que se desee construir (entre 30 y $280 \mathrm{~mm}$.), así como distintos diámetros (4 ó $5 \mathrm{~mm}$.) o pletinas $(2 \times 6 \mathrm{~mm}$.).

La albañilería armada regularmente con Murfor, es un "Nuevo material compuesto = La fábrica armada", que tiene capacidad para soportar tracciones, lo que le permite afrontar sin fisurarse ni agrietarse: (A) Deformaciones impuestas, (B) Flexión vertical y (C) Flexión horizontal. Segun se disponga el armado, se consigue el efecto de trabajo combinado que resuelve el conjunto de solicitaciones $[(A)+(B)+(C)]$ a la vez.

La triangulación de la cercha del material compuesto, ofrece una gran adherencia. Genera un efecto zuncho que traba bidireccionalmente en el plano del tendel, y aumenta la resistencia a compresión del muro. Garantiza la colocación precisa de los alambres en el tendel y permite una respuesta unitaria de la fábrica en todo su grueso, frente a solicitaciones.

El material compuesto fábrica armada, con cuantía mínima del $0.05 \%$ de su sección, regularmente repartida, previene su fisuración. Esto supone armar con Murfor los tendeles separados entre $40650 \mathrm{~cm}$ de altura, (Eurocódigo 6)(1).

Si se disponen varias armaduras Murfor agrupadas adecuadamente, aumentan las prestaciones técnicas de la albañilería, como es el caso de dinteles hechos con material de fábrica homogéneo, en muros de carga o cerramiento (2).

Al proyectar con las posibilidades que ofrecen las armaduras Murfor, se abre un campo de nuevas soluciones que simplifican y economizan la construcción. Esto permite que se potencie la Arquitectura de fábrica y mejore su calidad (3).

+ En zonas sísmicas, la fábrica armada disminuye los desperfectos en la albañilería y evita daños por rotura frágil

+ En rehabilitación, la fábrica armada aporta sencillas soluciones, adecuadas a la patología concreta de cada caso

Se ha elaborado un Manual (*), donde se exponen los campos de aplicación de la fábrica armada y los consejos de colocación. Contiene tablas de cálculo simplificado para los casos más habituales. (Existe también una guía práctica.)

1.- PREVIENE la FISURACION DE LA ALBAÑILERIA

- Frente a asientos diferenciales del terreno bajo muros de carga

- Frente a flexiones de vigas o forjados bajo tabiques y cerramientos

- Frente a contracción, retracción o dilatación de paños de fábrica de gran longitud

- Frente a concentración de tensiones, alrededor de huecos de puertas o ventanas

- Frente a concentración de tensiones, bajo cargas puntuales, variaciones de altura, grueso...

\section{2.- AUMENTA LAS PRESTACIONES TECNICAS}

- En el incremento de separación de las juntas de dilatación

- En la ejecución de dinteles de material de fábrica homogéneo

- En muros sometidos a la presión o succión del viento

- En muros sometidos al empuje del terreno, piscinas...

- En la ejecución de cadenas de enlace y reparto de forjados

3.- POTENCIA Y ECONOMIZA LA ARQUITECTURA

- Al evitar puentes térmicos, al no perder homogéneidad la fábrica con el armado

- Al construir muros dobles n capuchinos, de hojas de diversos materiales

- Al permitir dejar esperas que unan solidariamente diversas fases de obra, o muros pasantes

- Al poder diseñar sin la necesidad de aparejar las piezas de la albañilería

- Al incrementar la libertad técnica y formal del Arquitecto.

(*) MANUAL Murfor: "La fábrica armada" - Adell/Lahuerta - Bekaert. Tvra. Gràcia 30-3º D 08021 Barcelona. Tel:93/4140852 


\section{LA FÁBRICA ARMADA}

Murfor: La fábrica armada

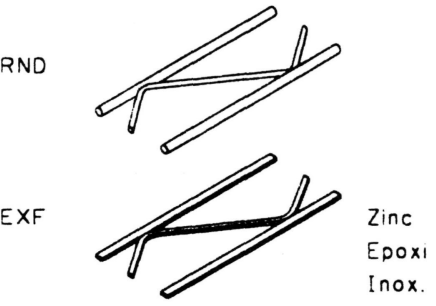

Armadura de tendel tipo Cercho

(A) Deformaciones impuestos
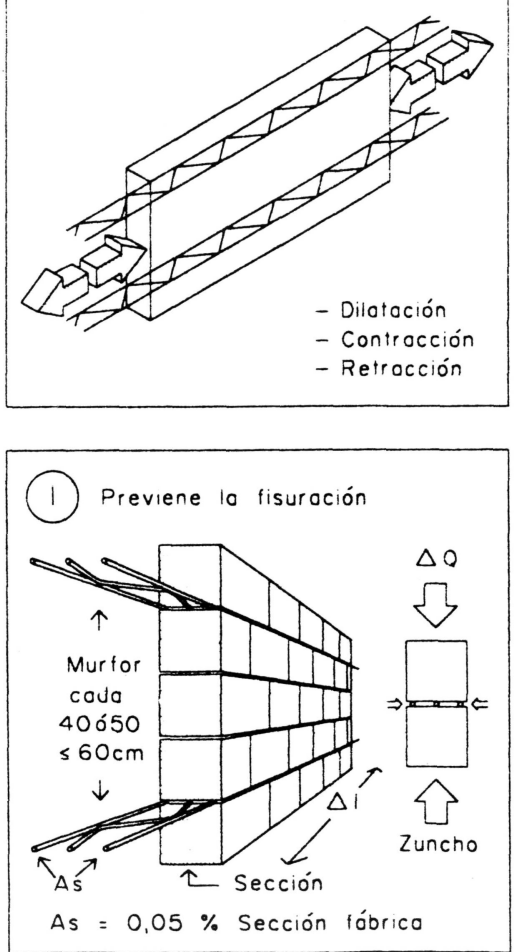

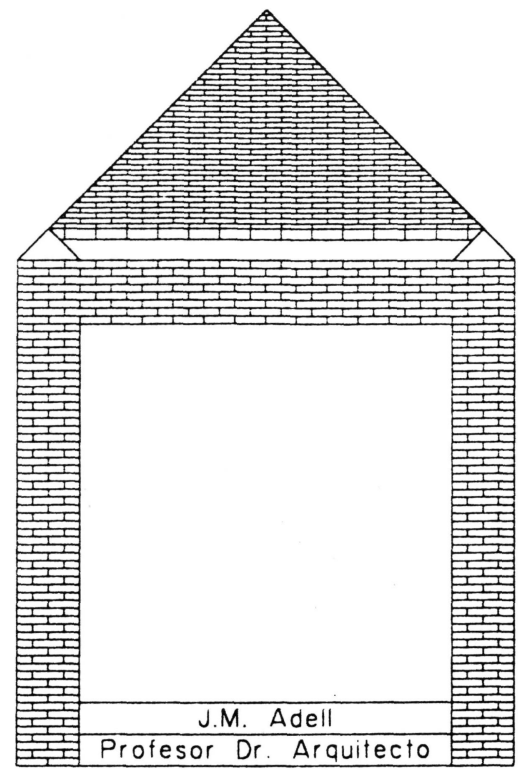

MATERIAL COMPUESTO

$\wedge \supset \equiv\lfloor L$

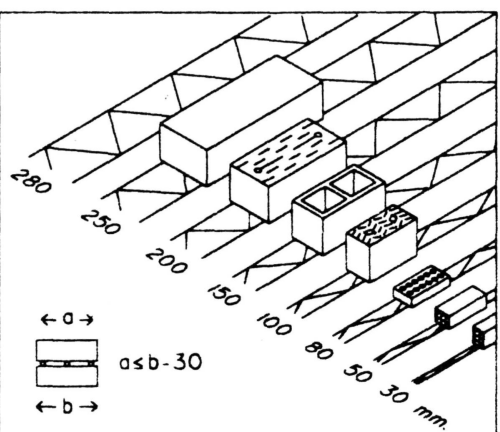

Hoy anchuras apropiodos poro cada material y tipo de fábrica

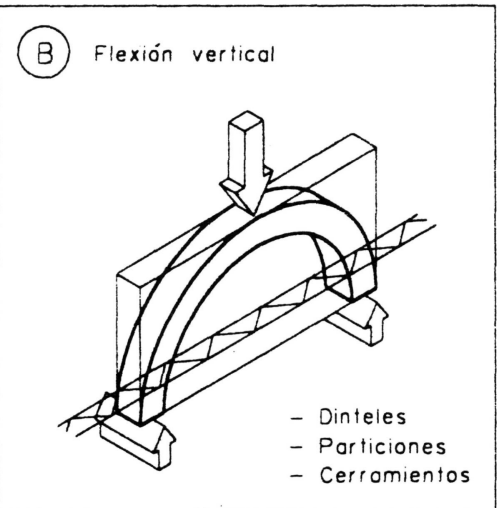

(2) Aumento los prestaciones técnicos

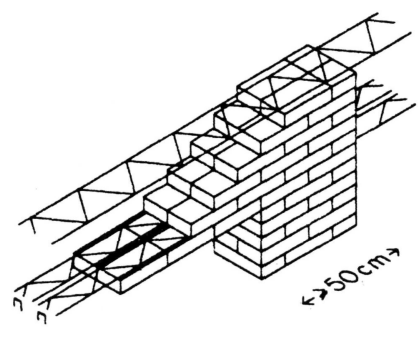

Ganchos Murfor LHK

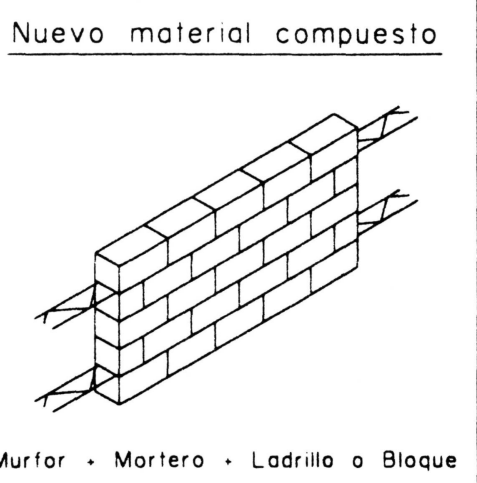

(C) Flexión norizonta

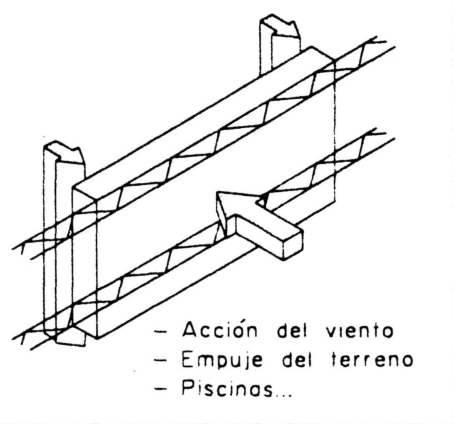

(3) Potencio y economizo la Arquitecturo

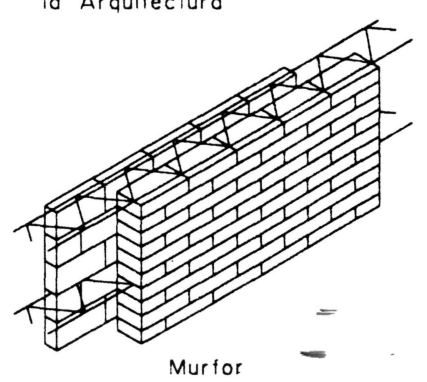

LA IDEA CONSTRUCTIVA 